\title{
PERFIL DA RESISTÊNCIA CARDIORESPIRATÓRIA EM MULHERES IDOSAS COM SOBREPESO DO PROGRAMA DE ATIVIDADE FÍSICA NO SESC DE NOVA FRIBURGO / RJ/ BRASIL
}

\author{
CARDIORESPIRATORY RESISTANCE PROFILE \\ IN ELDERLY WOMEN WITH OVERWEIGHT IN SESC OF NOVA \\ FRIBURGO/RIO DE JANEIRO - BRASIL OF PHYSICAL ACTIVITY PROGRAM
}

\author{
AUTORES \\ Helio Lemos Furtado ${ }^{1,3,4}$ \\ Fabio Dutra Pereira ${ }^{3,4}$ \\ Maria Helena Rodrigues Moreira ${ }^{2}$ \\ Estélio Henrique Martin Dantas ${ }^{1,3,4}$ \\ ${ }^{1}$ Universidade Castelo Branco \\ ${ }^{2}$ Universidade de Trás-os-Montes e Alto Douro \\ ${ }^{3}$ LABIM - Laboratório de Biociências \\ da Motricidade Humana - UCB \\ ${ }^{4}$ GDLAM - Grupo de Desenvolvimento \\ Latino-Americano da Maturidade \\ ${ }^{5}$ NEPE - Núcleo de Estudos e Pesquisas \\ do Envelhecimento
}

PERFIL DA RESISTÊNCIA

CARDIORESPIRATÓRIA EM

MULHERES IDOSAS COM

SOBREPESO DO PROGRAMA

DE ATIVIDADE FÍSICA NO SESC DE

NOVA FRIBURGO / RJ/ BRASIL

4(1): 21-26

PALAVRAS-CHAVE

resistência cardiorespiratória;

idosas; sobrepeso; atividade física

\section{KEYWORDS}

cardiorespiratory resistance;

elderly; overweight; physical activity

data de submissão

Setembro 2007

data de aceitação

Dezembro 2007
RESUMO

O objetivo do estudo foi investigar se um programa de atividade física promove melhoras significativas na capacidade cardiorespiratória de idosas. Participaram do estudo 71 gerontes do sexo feminino, sendo divididas em quatro grupos nas respectivas faixas etárias: G1 (60-64 anos; $n=29$ ), $G 2$ (65-69 anos; $n=22$ ), G3 (70-74 anos; $n=11$ ) e G4 (75-79 anos; $\mathrm{n}=09$ ). $A$ aptidão cardiorespiratória foi avaliada através do teste de caminhada de 6 minutos de Rikli e Jones (1998). Foi utilizado o teste Shapiro-Wilk, para verificação da gaussianidade da amostra, com nível de significância $<0,05$, utilizou-se o programa SPSS 10.0 for Windows. Analisando a variável distância percorrida, obteve-se as seguintes médias: G1 $=526 \mathrm{~m}, \mathrm{G2}=509,7 \mathrm{~m}, \mathrm{G3}=491,3 \mathrm{~m}$ e G4=479,4 m, pode-se concluir que somente o G1 não obteve seu valor dentro da faixa de referência. Considerando a possibilidade da não especificidade da prescrição do exercício físico para cada faixa etária apresentada pelos grupos estudados, o programa de atividade física realizado foi capaz de proporcionar a $60,5 \%$ daquelas idosas, um perfil satisfatório para a resistência cardio-respiratória.
ABSTRACT

The study objective was to investigate if one physical activity program promotes significant improvements in the cardiorespiratory capacity in elderly. They had participated of study $\mathbf{7 1}$ feminine elderly people, being divided in four groups in the respective age band: G1 [60-64 years old; $n=29$ ), $G 2$ (65-69 years old; $\mathrm{n}=22)$, G3 (70-74 years old; $\mathrm{n}=11$ ) and $\mathrm{G} 4$ (75-79 years old; $n=09$ ). The cardiorespiratory fitness was evaluated through the 6 minutes walked test (Rikli and Jones 1998). After that, for normality verification had been used the Shapiro-Wilk test, with significance level $<0,05$, was used program SPSS 10,0 for Windows. Analyzing the covered distance changeable, one got the following averages: $\mathrm{G} 1=526 \mathrm{~m}, \mathrm{G2}=\mathbf{5 0 9 , 7} \mathrm{m}$, $\mathrm{G} 3=491,3 \mathrm{~m}$ and $\mathrm{G} 4=479,4 \mathrm{~m}$, can be concluded that the $\mathbf{G 1}$ did not only get its value inside of the reference band. Considering the possibility of not the specific of the prescription physical exercise for each age band presented by the studied groups, the physical activity program was capable to provide $60.5 \%$ elderly, a satisfactory profile for the cardio-respiratory resistance. 


\section{INTRODUC̣ÃO}

0 aumento da expectativa de vida ocorrido ao longo dos últimos anos, devido principalmente, aos avanços da medicina e da produção mundial de alimentos, tem permitido um gradual e progressivo envelhecimento da população mundial ${ }^{5}$.

No Brasil, este fenômeno trouxe significativas mudanças no âmbito social e econômico. Uma das preocupações sociais sobre o envelhecimento gira em torno da capacitação funcional e o envelhecimento pode causar algum tipo de incapacidade funcional nos 22,2 anos de expectativa de vida a partir dos 60 anos de idade ${ }^{4,8}$

0 fato de muitos gerontes não conseguirem manter sua independência nas atividades da vida diária (AVD), faz com que haja comprometimento de sua qualidade de vida ${ }^{13,15}$. Das alterações funcionais oriundas do envelhecimento, destaca-se o aumento da prevalência de doenças cardiovasculares e neste sentido, o Ministério da Saúde do Brasil alerta que $33 \%$ dos óbitos ocorrem de patologias desta natureza ${ }^{3,19}$. Um dos aspectos que pode colaborar para esta situação é o sedentarismo, condição que normalmente acompanha o envelhecimento. Por este fato, um programa de atividade física poderia combater o sedentarismo e prevenir tais patologias, uma vez que, os exercícios físicos aumentam a potência aeróbica entre $10-40 \%$, especialmente pelo incremento da diferença arteriovenosa de oxigênio, volume sistólico, débito cardíaco, volume plasmático e sanguíneo ${ }^{10,11}$.

Considerando toda a complexidade do processo de envelhecimento e as possibilidades benéficas da prática da atividade física, este estudo pretende verificar o perfil do condicionamento cardiorespiratório em mulheres idosas do programa de atividade física no SESC de Nova Friburgo/RJ.

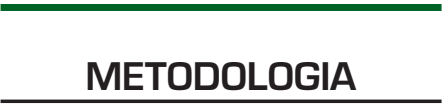

Amostra

O estudo contou com uma amostra constituída de $\mathbf{7 1}$ gerontes do sexo feminino, residentes no município de Nova Friburgo/RJ. Sendo divididas em quatro grupos sendo as respectivas faixas etárias: G1 (6064 anos; $n=29$ ), $G 2$ (65-69 anos; $\mathrm{n}=22$ ), G3 (70-74 anos; $\mathrm{n}=11$ ) e G4 (75-79 anos; $n=09$ ). Como critério de inclusão, todas as idosas deveriam estar com no mínimo de $\mathbf{3}$ meses de prática regular ${ }^{2}$.

Neste sentido, foram submetidas a um programa de atividade física composto de uma freqüência de três seções semanais, constituída de 5 minutos de aquecimento, na parte principal da aula 20 minutos eram destinados a atividades aeróbicas e outros 20 minutos a atividades neuromusculares (duas por semana treinamento de força e uma vez treinamento de flexibilidade) na volta à calma eram realizados 5 minutos de alongamento. Foi considerado como critério de exclusão qualquer tipo de condição patológica aguda ou crônica que pudesse comprometer ou que tornasse um fator de impedimento para realização do teste de caminhada de seis minutos ${ }^{17}$.

As participantes desta pesquisa assinaram o termo de consentimento e os procedimentos experimentais foram executados dentro das normas éticas previstas na Resolução n. 196, de 10 de Outubro de 1996, do Conselho Nacional de Saúde.

0 estudo teve seu projeto de pesquisa submetido e aprovado pelo
Comitê de Ética em Pesquisa Envolvendo Seres Humanos da Universidade Castelo Branco, RJ.

\section{Procedimentos}

Como caráter reprodutivo, a amostra deste estudo foi submetida ao referido teste, que propôs às avaliadas, a caminhar tão rápido possivel (sem correr] quanto a maior distância em 6 minutos um percurso de 45,72 metros, dividido em 10 segmentos de 4,57 metros e demarcado com cones e fita crepe. A execução do teste se deu em um local aberto, com superfície não derrapante e nivelada, além de ter $23,7^{\circ} \mathrm{C}$ como temperatura ambiente no dia da avaliação. Para determinar a distância percorrida, uma ficha plástica foi dada às participantes toda vez que passavam pelo cone controle ou ainda quando um avaliador ou ajudante marcava a volta completada. Se fosse necessário, as avaliadas poderiam parar e descansar (em cadeiras disponíveis) e depois continuar caminhando ${ }^{17}$.

Visando assegurar a homogeneidade da amostra, realizou-se a mensuração e classificação do estado nutricional através do calculo do Índice de Massa Corporal (IMC) utilizando-se uma balança com estadiômetro (Filizola, Brasil). Adotou-se como referência para as variáveis, estado nutricional (IMC) e distância percorrida, os valores predistos ${ }^{6,17}$.

\section{Estatística}

Foram realizadas as técnicas de estatística descritiva (mediana, média, desvio padrão e coeficiente de variação) no sentido de enquadramento dos grupos estudados nos índices referencia. Para verificar a distribuição da amostra foi empregado o teste Shapiro-Wilk com nível de significância <0,05. Em ambos cálculos estatísticos utilizou-se o programa SPSS 10.0 for Windows. 


\section{RESULTADOS}

Os resultados do presente estudo serão apresentados na tabela 1 e 2 , e figuras 1 e 2 que mostrarão a mediana, média, desvio padrão, coeficiente de variação e distribuição das variáveis: estado nutricional (IMC) e distância percorrida, para os quatro grupos estudados.

Analisando a tabela 1 e 2 , pode-se observar que os grupos 1 e 3 apresentam distribuição próxima da normalidade para todas as variáveis, fato não ocorrido no G2 e G4. A rejeição da normalidade apresentada pelos referidos grupos não comprometeu a análise dos resultados, uma vez que foi utilizada a média das variáveis para todos os grupos, pois o CV\% se apresentou inferior a $25 \%{ }^{18}$.

Assim ao enquadrar a média do IMC dos grupos com os de referência deve-se considerar que todos os grupos apresentam a classificação do estado nutricional (IMC) como sobrepeso. Quanto a variável distância percorrida pelos grupos, o enquadramento se deu nas respectivas faixas etárias e desta feita, é correto afirmar que somente o $\mathrm{G1}$ não obteve seu valor dentro da faixa de referência. 0 gráfico subseqüente expressa esta afirmativa.

\section{DISCUSSÃO}

Um ponto a ser mencionado neste momento é a utilização do IMC na aplicação da avaliação do estado nutricional de idosos. Atualmente, recomendam-se como pontos de corte para definição de baixo peso (IMC $<22 \mathrm{~kg} / \mathrm{m}^{2}$ ), peso normal (IMC entre 22 a $27 \mathrm{~kg} / \mathrm{m}^{2}$ ) e sobre-peso (IMC $>27 \mathrm{~kg} / \mathrm{m}^{2}$ ] que diferem dos valores recomendados para adultos.

\begin{tabular}{cccccccccc} 
& & & & & & & \multicolumn{3}{c}{ Shapiro-Wilk } \\
\cline { 8 - 10 } Variável & Grupo & Mediana & Média & $\begin{array}{c}\text { Desvio } \\
\text { Padrão }\end{array}$ & CV\% & Statistic & Df & $\begin{array}{c}\text { Significance } \\
\text { (p) }\end{array}$ \\
\hline \multirow{4}{*}{ IMC } & 1 & 29,00 & 29,4 & 1,8 & 6,20 & 0,927 & 29 & 0,055 \\
& 2 & 28,5 & 29,0 & 2,1 & 7,49 & 0,832 & 22 & $0,010 *$ \\
& 3 & 29,00 & 29,4 & 1,8 & 6,14 & 0,886 & 11 & 0,160 \\
& 4 & 28 & 28,5 & 1,0 & 0,03 & 0,893 & 09 & 1,279 \\
\hline
\end{tabular}

TABELA1

IMC para os grupos estudados.

* Valores de significância $\mathrm{p}<0,05$ rejeitam a normalidade da distribuição.

Esta proposta leva em consideração as mudanças na composição corporal que ocorrem com o enveIhecimento. Mesmo não expressando tais mudanças, a facilidade de obtenção de dados de peso e estatura bem como, sua boa correlação com morbidade e mortalidade, justifica a utilização do (IMC) em estudos epidemiológicos e na prática clínica desde que se usem pontos de corte específicos para a idade $^{6}$.

0 presente estudo demonstrou em seus resultados que todos os quatro grupos apresentaram a classificação do estado nutricional como sobrepeso. Corroborando com este achado, ao estudar 129 muIheres com idade média de 63,9 anos, buscando relacionar a adiposidade corporal e a idade com a capacidade funcional para realizar as atividades da vida diária. Para isto, utilizou além da adipometria, o IMC e neste, o valor médio apresentado foi de 27,5 sendo também classificado como sobrepeso ${ }^{16}$.

Uma outra pesquisa que vem confirmar as tendências do presente estudo, verificou os efeitos de um programa de orientação de atividade física e nutricional sobre a ingestão alimentar e composição corporal de mulheres fisicamente ativas. Assim realizaram um pré-teste em 64 mulheres com idade média de 61,9 anos, onde estas também foram divididas em quatro

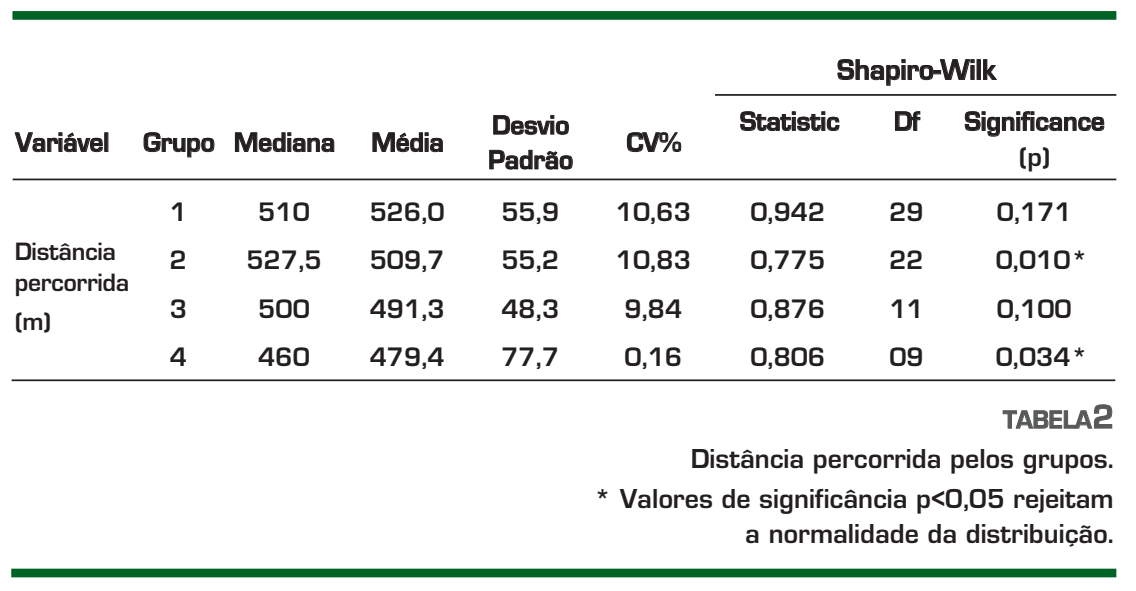




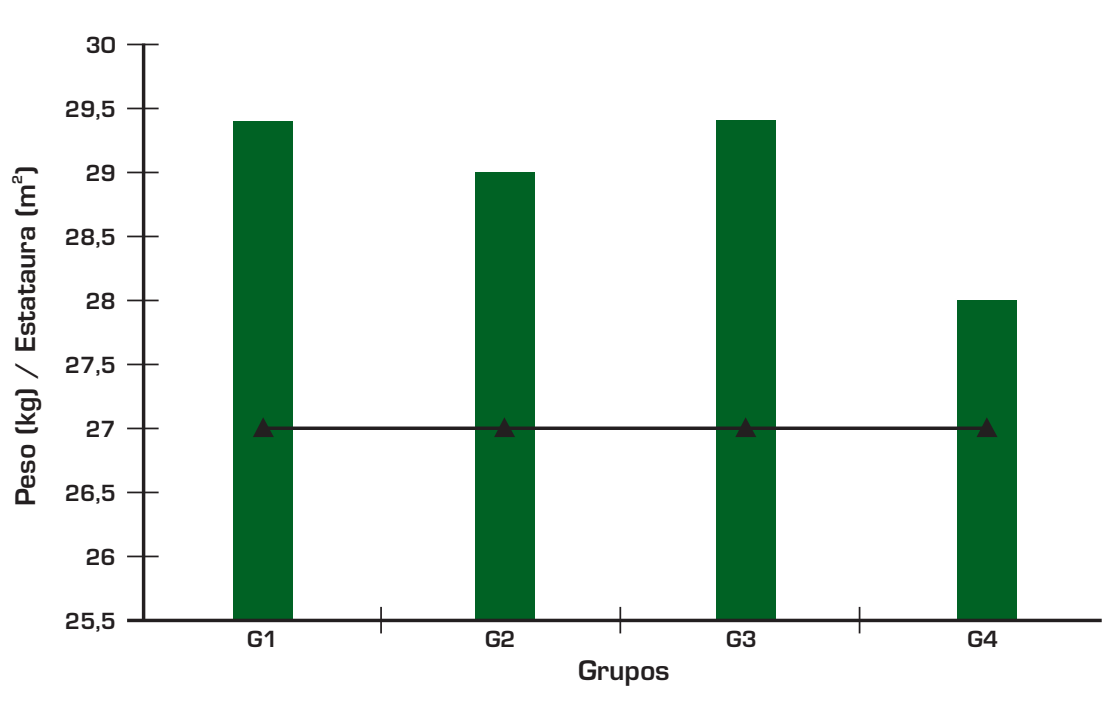

FIGURA 1

IMC dos grupos.

LEGENDA

IMC dos grupos $\quad-$ IMC predisto [sobrepeso] grupos e apresentaram os seguintes resultados para o estado nutricional: $\mathrm{G1}$ (IMC=27,3), G2 (IMC= 28,1), G3 (IMC=26,9) e $G^{\text {Controle }}$ (IMC=27,2). Podendo ser concluído que mesmo o G3 apresentando o valor de 0,1 inferior ao índice de classificação de sobrepeso este grupo foi o único que assim não se classificou?
Ainda focado no estado nutricional, porém discordam com o presente estudo, ao verificar os efeitos de um programa de atividades físicas e educacionais para idosos sobre o desempenho em testes de atividades de vida diária, avaliaram 15 idosas com idade entre 61-77 anos e encontraram como estado nutricional do pré-teste um valor médio

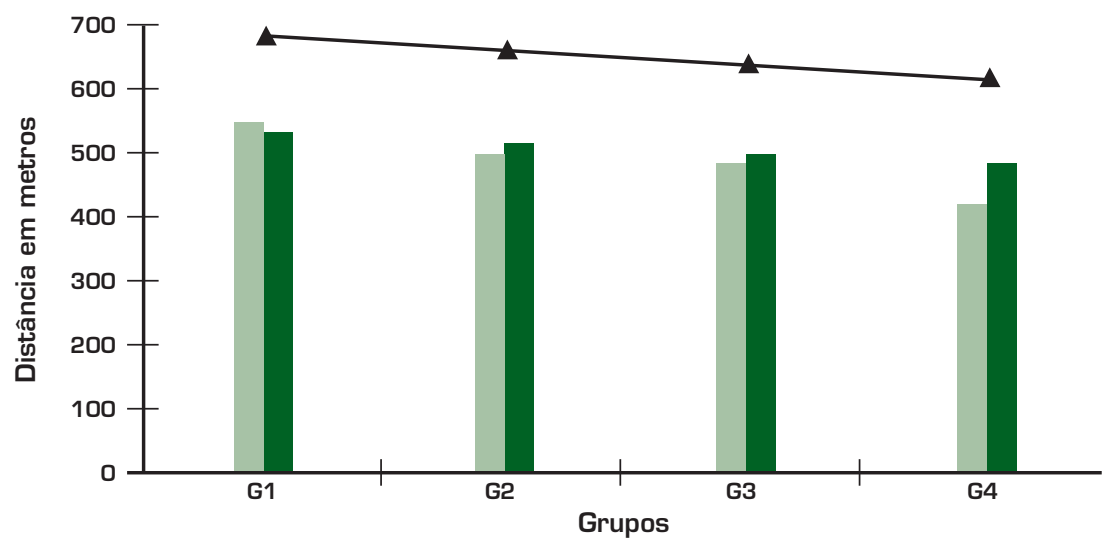

FIGURA?

Distância percorrida em 6 minutos.

LEGENDA Dist. percorrida $\square$ Dist. mínima (R\&J) - - Dist. máxima [R\&J] de IMC de 25,5 sendo classificado como peso normal ${ }^{9}$.

Quanto à resistência cardiorespiratória, o G4 foi o único grupo que apresentou um valor consistente para a distância percorrida no teste proposto, isto porque o presente estudo demonstrou que o G1 apresentava os valores da distância percorrida em seis minutos inferior aos valores normativos ${ }^{17}$. Isso pode ter ocorrido uma vez que ao prescrever as atividades aeróbias os responsáveis por tal prescrição tenham se baseado como caráter de segurança na idade mais elevada das participantes. Esta suposição se apóia na recomendação que a intensidade da fase aeróbia de um treinamento pode ser determinada através do percentual do consumo máximo de oxigênio $\left[\mathrm{VO}_{2} \mathrm{máx}\right.$ ou da freqüência cardíaca máxima [FCmáx] efetivamente estabelecidos em um teste ergométrico ou estimados através de fórmulas. Geralmente, é recomendada uma intensidade moderada, como 40 a $75 \%$ do $\mathrm{VO}_{2}$ máx ou 55 a $85 \%$ da FC máxima $^{14}$. Deve-se observar que sessões com intensidade alta podem estar associadas a um maior risco de desistência, devido a desconforto muscular, especialmente nas fases iniciais de um programa de exercícios. Por este fato o programa de treinamento possivelmente tenha subestimado a zona de treinamento de G1. Os grupos 2 e 3 apresentaram os valores da distância percorrida bem próximo do limite inferior estabelecido ${ }^{17}$, corroborando com esta hipótese.

Outro estudo que buscou analisar os efeitos de um programa de atividade física na capacidade aeróbia de mulheres idosas, estudou uma amostra que se constituiu de 20 mulheres, na faixa etária de 60 a 64 anos (média de 62,55 anos), 
praticantes de um programa de atividade física por pelo menos três meses. Para tal, também foi utilizado o teste de caminhada de seis minutos ${ }^{17}$. Em seu resultado foi encontrado o valor médio de 544,1 metros para a distância percorrida em 6 minutos. Quando comparado ao valor predito do protocolo observou-se que este achado não incidiu nos valores normativos para a referida faixa etária. Este fato pode se justificar porque o programa de atividade física realizado era composto de atividades lúdicas, relaxamentos, reflexos neuromusculares e aeróbios, mas sem enfatizar este componente ${ }^{12}$.

0 presente estudo, por ser descritivo, limita-se a sugerir a razão dos resultados do G4 no teste de caminhada de 6 minutos em relação aos outros grupos, muito embora o programa de treinamento descrito em sua metodologia substancie tal sugestão. Uma pesquisa que torna esta sugestão bastante consistente buscou avaliar a influência da hidroginástica sobre a aptidão física de idosas. Neste sentido, estudou-se 37 mulheres sedentárias, com idade média de 78,0 anos, que foram submetidas a um pré-teste de caminhada de 6 minutos, obtendo um resultado médio de 419,9m. Valor este abaixo dos preditos ${ }^{17}$. Após um programa de atividade física (Hidroginástica) composto de 5 minutos de aquecimento, 20 minutos de componente aeróbio, 20 minutos de neuromuscular e 5 minutos de relaxamento que perdurou por 3 meses com freqüência de duas sessões semanais. Os resultados do pós-teste da amostra foram de $513 \mathrm{~m}$, apresentando uma diferença de significância de $p<0,05$ e podendo ser enquadrado na faixa de normalidade dos valores preditos ${ }^{1}$.
Em conclusão, mesmo considerando a possibilidade da não especificidade da prescrição do exercício físico para cada faixa etária apresentada pelos grupos estudados, o programa de atividade física realizado no SESC/Nova Friburgo foi capaz de proporcionar a $60,5 \%$ daquelas idosas, um perfil satisfatório para a resistência cardiorespiratória.

\section{CORRESPONDÊNCIA}

Helio Lemos Furtado

Rua Coronel João Olintho, n 271, Recreio dos Bandeirantes, CEP- 22790-170

Rio de Janeiro, Brasil

REFERÊNCIAS

1. Alves RV, Mota J, Costa MC, Alves JGB (2004). Aptidão física relacionada à saúde de idosos: influência da hidroginástica. Rev Bras Med Esporte 10:31-37.

2. American College Of Sports Medicine (1998). Position stand: the recommended quantity and quality of exercise for developing and maintaining cardiorespiratory and muscular fitness, and flexibility in healthy adults. Med. Sci. Sports Exerc 30:975-991.

3. Benseñor IM, Lutofo PA (2002). Estado atual do tratamento e controle de diabetes melito, da dislepidemia e da hipertensão arterial no Brasil e no mundo: condutas gerais para a adequada manutenção do controle. In: Mion JRD, Nobre F. Risco cardiovascular geral. São Paulo: Lemos editorial, 17-43.
4. Camargos MCS, Perpétuo IHO, Machado CJ (2005). Expectativa de vida com incapacidade funcional em idosos em São Paulo. Rev. Panam. Salud Publica/Pan Am J Public Health 17:359-386.

5. Cantera IR. Envejecimiento, siglo XXI y solidadidad (2002). Rev. Esp. Geriatr. Gerontol 37:3-6.

6. Cervi A, Franceschini SCC, Priore SE (2005). Análise crítica do uso do índice de massa corporal para idosos. Rev. Nutr. 18:765-775.

7. Ferreira M, Matsudo S, Matsudo V, Braggion G (2003). Efeito de um programa de atividade física e nutricional sobre a ingestão alimentar e composição corporal de mulheres fisicamente ativas de 50 a 72 anos de idade. Rev. Bras. Cienc. Mov 11: 35-40.

8. Francisco PMSB, Donalisio MRC, Lattorre MRDO (2003). Tendência da mortalidade por doenças respiratórias em idosos do Estado de São Paulo, 1980 a 1998. Rev Saúde Pública 37:191-196.

9. Hernandes ESC, Barros JF (2004). Efeitos de um programa de atividades físicas e educacionais para idosos sobre o desempenho em testes de atividades da vida diária. Rev. Bras. Cienc. Mov 12:43-50.

10. Matsudo SM (2001). Evolução da aptidão física e capacidade funcional de mulheres ativas acima de 50 anos de idade de acordo com a idade cronológica - Tese de Doutorado. São Paulo, Universidade Federal de São Paulo. Escola Paulista de Medicina.

11. Matsudo SM, Matsudo VKR, NetoTLB (2000). Impacto do enveIhecimento nas variáveis antropométricas, neuromotoras e metabólicas da aptidão física. Rev. Bras. Cienc. Mov. 8:15-21. 
12. Miranda EP, Rabelo TH (2006). Efeito de um programa de atividade física na capacidade aeróbia de mulheres idosas. Movimentum 1.

13. Mussoll J, Espinosa MC, Quera D, Serra ME, Pous E, Villar-Roya (2002). Resultados de la aplicación em atención primaria de um protocolo de valoración geriátrica integral em ancianos de riesgo. Rev. Esp. Geriatr. Gerontol 37:249-253.

14. Nóbrega ACL, Freitas EV, Oliveira MAB, Leitão MB, Lazzoli JK, Nahas RM (1999). Posicionamento oficial da Sociedade Brasileira de Medicina do Esporte e da Sociedade Brasileira de Geriatria e Gerontologia: atividade física e saúde no idoso. Rev Bras Med Esporte 5:207-211.

15. Pine MJ, Murphy AJ, Watsford ML (2005). Role of respiratory system function in the age-related decline of human functional capacity. Australasian Journal on Ageing 24:153-156.

16. Raso WA (2002). Adiposidade corporal e a idade prejudicam a capacidade funcional para realizar as atividades de vida diárias de mulheres acima de 47 anos. Rev Bras Med Esporte 8.

17. Rikli RE, Jones CJ (1998). The Reliabiltiy and Validity of a 6-Minute Walk Test as a measure of Physical Endurance in Older Adults. Journal of Aging and Physical Activity 6.

18. Shimakura SE (2005). Coeficiente de Variação. Disponível em <http://www.est.ufpr.br/ silvia/ CE055/node26.html.> Acesso em 14 Out. 2005.

19. Yanowitz FG, La Monte AM (2002). Physical activity and health in the elderly. Curr sport med resp 1:354-361.

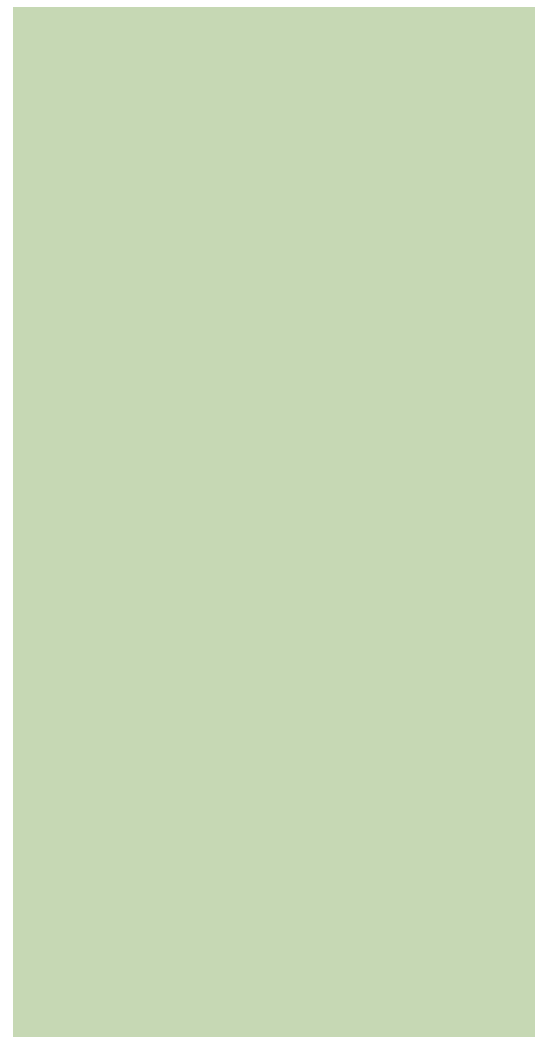

